\title{
Prácticas de autocuidado y la adherencia al TARGA en personas que viven con VIH en un hospital nacional de Lima
}

\author{
Meneses-La Riva Mónica'; Mayorca-Carmelo Carmen²
}

\section{RESUMEN}

Objetivo: determinar la relación entre las prácticas de autocuidado y la adherencia al TARGA en personas que viven con VIH de un hospital nacional. Material y métodos: estudio cuantitativo, correlacional, de corte transversal, la muestra estuvo compuesta de 288 personas que viven con VIH; se elaboró un cuestionario de 37 preguntas sobre prácticas de autocuidado y adherencia al TARGA, las cuales fueron validadas a través de una juicio de expertos y una prueba piloto; posteriormente, se obtuvo un listado de personas que viven con VIH de la historia clínica. Resultados: La recolección de datos permitió obtener los resultados que muestran que las prácticas son inadecuadas en su mayoría $(99,7 \%)$, solo presentan prácticas de autocuidado adecuadas 0,3\%. El perfil de adherencia, en su mayoría, también son no adherentes en un $70,1 \%$, mientras que $29,9 \%$ son adherentes. El coeficiente de correlación rho de Spearman $(\mathrm{R}=0,445)$ muestra una correlación significativa $(\mathrm{p}=0,000)$, la cual resultó positiva y de nivel medio, lo que significa que niveles bajos de prácticas de autocuidado se corresponden con niveles bajos de adherencia, y viceversa. Conclusiones: las prácticas de autocuidado y la adherencia al tratamiento del TARGA se encuentran relacionadas con la decisión personal de lograr la madurez en su propio autocuidado, esto quiere decir que los patrones del comportamiento están interactuados según su estilo de vida.

Palabras clave: adherencia al TARGA, Autocuidado, VIH. Perú. (Fuente DeCs BIREME).

\section{Self-care practices and adherence to TARGA in people living with HIV at a national hospital Lima}

\begin{abstract}
Objetive: To determine the relation between the practices of auto care and the adherence to the TARGA in persons who live with HIV of a National Hospital. Material and Methods: quantitative Study, correlacional, of transverse court, sample of 288 persons who live with HIV; there was elaborated a questionnaire of practices of auto care and adherence to the TARGA of 37 questions, which were validated across one experts' and a pilot test was realized and later obtained a persons' list through that they live with HIV of the clinical history. Results: The compilation of information allowed to obtain the results that show us that the practices are inadequate in the main with $99.7 \%$, only they present suitable practices of auto care $0.3 \%$. The profile of adherence in the main also adherents are not in 70.1 $\%$ and $29.9 \%$ adherents. The coefficient of correlation Rho de Spearman $(\mathrm{R}=0.445)$ shows a statistically significant correlation ( $\mathrm{p}=0.000$ ), which turned out to be positive and of average level, which means that low levels of practices of auto care correspond with low levels of adherence and vice versa. Conclusions. The practices of auto care and the adherence to the treatment of the TARGA are associated with the personal decision to achieve the maturity in his own auto care; this wants to say that the bosses of the behavior are interacted according to his way of life.
\end{abstract}

Key words: Adherence to the TARGA, Auto care, HIV. Peru. (Source DeCs BIREME).

Magíster en Enfermería. Escuela de Enfermería- Universidad Cesar Vallejo. Lima, Perú

Magíster en Enfermería. Hospital Nacional Arzobispo Loayza. Lima, Perú. 


\section{INTRODUCCIÓN}

El virus de la inmunodeficiencia humana (VIH) infecta a las células del sistema inmunitario y altera o anula su función, esta infección produce un deterioro progresivo del sistema inmunitario, con la consiguiente «inmunodeficien cia». Asimismo, se considera que el sistema inmunitario es deficiente cuando deja cumplir su función de lucha contra las infecciones y enfermedades.

El síndrome de inmunodeficiencia adquirida es considerado como un problema de salud pública y de gran naturaleza debido a la magnitud de los daños que se evidencian en la morbilidad y mortalidad de la población (1). En el mundo, residen más de 35,3 millones de personas que están infectadas por el VIH, de las que 2,1 millones son adolescentes (de 10 a 19 años); además, la gran mayoría de las personas infectadas por el VIH, viven en países de ingresos bajos y medios, y se estima que en el año 2012, unos 2,3 millones de personas que contrajeron dicha infección. A esta fecha, el Perú tenía unos 76000 portadores del VIH y frente este contexto, es preciso promover un tratamiento integral especializado aunado a un equipo multidisciplinario de salud que afronte dicha enfermedad, considerando que los obstáculos existentes que afronta el paciente con VIH, parten de la no aceptación de la propia enfermedad, seguido de la falta de apoyo de la familia, entre otros. Además, es menester reconocer que hay factores políticos (indiferencia o despreocupación, interferencia frente al libre flujo de información), socioculturales (normativas, roles, tabúes) y económicos (pobreza, falta de recursos, desigualdad de ingresos) que inciden directamente en el problema de la infección con $\operatorname{VIH}(1,2)$.

Un gran avance y conveniente para el control de la infección por VIH, ha sido el descubrimiento del tratamiento antirretroviral de gran actividad (TARGA) y su trayectoria universal. Asimismo, el Ministerio de Salud de Perú (MINSA) inició la provisión del TARGA desde mayo de 2004, a partir de entonces, ha sido posible incrementar su cobertura, optimizando la calidad de vida de las personas que viven con VIH y retrasando la progresión de la infección a estadios evolutivos e incipientes, donde el Estado se esfuerza en consolidar el programa del TARGA (3).

El MINSA cuenta con el Servicio de Infectología, este programa realiza un conjunto de actividades dirigidas a la población de todas las edades, con la finalidad de detectar precozmente los grupos de riesgo y disminuir la transmisión vertical y sanguínea. Entre las actividades que realiza este programa, se encuentra el tamizaje, despistaje de $\mathrm{VIH}$, despistaje de sífilis, confirmatoria de VIH, consejería pretest, consejería postest, médico ITS-VIH, control social, visitas domiciliarias, educación focalizada y grupal, que acompañan al tratamiento del TARGA (4).

La función primordial del enfermero(a) es el cuidado de las personas en el proceso salud enfermedad, así como generar conciencia en los sujetos con TARGA, destacan entre estas: la preparación de la primera consulta, entrevistas motivacionales, educación para la salud, consulta de adherencia y, en muchas ocasiones, el personal de enfermería actúa como referente cercano del paciente y como puente o enlace entre el paciente y el resto del equipo sanitario. Las actividades del enfermero en el proceso de cuidados al paciente con TARGA permiten refrescar los resultados de la terapia y es plenamente complementario a la función de los facultativos y del resto del equipo sanitario $(5,6)$. De este modo, la enfermera suscita que la persona se empodere de su propio autocuidado, para que contribuya en su calidad de vida. El autocuidado es importante para el desarrollo humano en la vida cotidiana y para el manejo de la enfermedad del VIH, dado que es una práctica que involucra el crecimiento personal y el desarrollo del logro de la madurez para asumir la enfermedad; asimismo, estimula a que sus prácticas saludables sean cotidianas para mejorar el estilo de vida (5).

La persona debe reconocer que el autocuidado debe ser cotidiano, porque su fomento y propagación, ayudará a reducir los efectos negativos de la enfermedad, no obstante, el paciente es consciente que se halla vulnerable y que la no adherencia al tratamiento es un obstáculo que lo afecta en lo económico, social y, sobre todo, en su calidad de vida. Igualmente, la adherencia al tratamiento es la capacidad de implicarse correctamente al cumplimiento riguroso del tratamiento, con el objetivo de conseguir el control del VIH. Los equipos multidisciplinarios de salud deben comprender y reconocer a la persona que vive con $\mathrm{VIH}$, en toda sus dimensiones humanas, registrando, valorando su unicidad y singularidad humana, todo ello para comprender la doble vertiente de la enfermedad y que el paciente tiene que lidiar diariamente, por otro lado, hacer frente a las exigencias que conllevan el cumplimiento estricto del tratamiento y la practicas de autocuidado, para evitar las enfermedad oportunistas. Por lo demás, el trabajo del equipo de salud multidisciplinario, debe ser un permanente intercambio de conocimientos científico-técnicos, humanísticos, de experiencias y vivencias.

Hoy, quienes acceden a la medicación y se adhieren responsablemente al tratamiento tienen la posibilidad de vivir existencias plenas y prolongadas. Por tanto, la adherencia otorga la posibilidad de vivir dignamente, con calidad y 
mejorando sustancialmente sus expectativas de vida; recapacitando que las prácticas de autocuidado son parte esencial de la adherencia y que exigen condiciones que las perfeccionen y se promuevan diariamente, a través de una terapia integral.

En conclusión, sida es un problema universal y de trascendencia social, es inevitable involucrar a la persona, a la familia y la comunidad; es necesario preservar las prácticas de autocuidado así como la buena actuación de las facilitadoras para la adherencia, todo ello favorece el comportamiento de cumplimiento del TARGA. El déficit de autocuidado puede ser obstáculos para la no adherencia, porque coloca a la persona enferma en situación de vulnerabilidad.

Esta investigación estudia las prácticas del autocuidado y la adherencia al TARGA de personas que viven con VIH y que participan en el programa en el Servicio de Infectología de un hospital de Lima.

\section{MATERIAL Y MÉTODOS}

Estudio cuantitativo, descriptivo correlacional y transversal, se describen las características de las variables y se hace un corte en el tiempo para identificar las prácticas del autocuidado que tiene la persona que vive con el VIH y la adherencia al TARGA; se realizó en los meses de octubre - diciembre de 2013 en un hospital nacional, situado en el departamento de Lima, en una población de 1543 personas mayores de 18 años de edad. Participaron en el estudio 288 pacientes del Servicio de Infectología, el cálculo del tamaño muestral se obtuvo aplicando formula estadística, posteriormente, se realizó un sorteo para iniciar el punto de partida de donde se inició la selección de los pacientes requeridos en la muestra.

El instrumento cuenta con 37 preguntas dicotómicas, su aplicación se divide en los siguientes segmentos: primer segmento: se elaboró un instrumento que fue validado por juicio de expertos, consta de datos sociodemográficos y fuentes de verificación. Segundo segmento: se aplica el instrumento, el cual contiene 29 puntos sobre prácticas de autocuidado y 8 sobre la adherencia al tratamiento de TARGA; presenta dimensiones como la alimentación, la higiene, la sexualidad, el apoyo emocional y la actividad física. Las respuestas afirmativas obtienen un punto y la respuestas negativas 0 puntos, excepto las preguntas 10 , 15,16 y 17 , que tienen un puntaje de 1 .

Se aplicó el cuestionario previo consentimiento informado a personas abordadas en consultorios o cuando les tomaban la muestra de CD4 o carga viral, luego se les orientó sobre el objetivo y el procedimiento de llenado del cuestionario. Para la validación del instrumento se consideró a diez expertos, quienes evaluaron la validez del contenido, el constructo y el criterio de los ítems, luego, los datos se ingresaron a una base de datos en el programa de Excel; posteriormente, esta base se transfirió al programa SPSS v15 para Windows Xp. (Statistical Product and Service Solutions) para el análisis correspondiente.

Para el análisis se utilizó el coeficiente de Alken, el estadístico Kaiser-Meyer Olkin; la confiablidad del instrumento se evaluó a través de una muestra piloto conformada por 30 personas que viven con VIH; finalmente, se obtuvo el coeficiente alfa de Cronbach 0,76.

\section{RESULTADOS}

Tabla 1. Prácticas de autocuidado de personas que viven con VIH adscritos en el programa de un hospital nacional. Octubre-diciembre 2013, Lima-Perú

\begin{tabular}{lcc}
\hline Prácticas de autocuidado & n & \% \\
\hline Prácticas adecuadas & 1 & 0,3 \\
Prácticas inadecuadas & 287 & 99,7 \\
Total & 288 & 100,0 \\
\hline
\end{tabular}

Tabla 2. Prácticas de autocuidado en personas que viven con VIH de un hospital nacional según las dimensiones. Octubre-diciembre 2013, Lima-Perú

\begin{tabular}{llccc}
\hline \multirow{2}{*}{$\begin{array}{l}\text { Dimensiones de prácticas } \\
\text { autocuidado }\end{array}$} & \multicolumn{2}{c}{ Prácticas adecuadas } & Prácticas & \% \\
& n & \% & inadecuadas & \% \\
\hline Prácticas de alimentación & 28 & 9,7 & 260 & 90,3 \\
Prácticas de higiene & 17 & 5,9 & 271 & 94,1 \\
Prácticas de sexualidad & 59 & 20,5 & 229 & 79,5 \\
Prácticas de apoyo emocional & 43 & 14,9 & 245 & 85,1 \\
Prácticas de actividad física & 86 & 29,9 & 202 & 70,1 \\
\hline
\end{tabular}


En la tabla 2, las prácticas de autocuidado son inadecuadas en porcentajes altos, destaca la de higiene con $94,1 \%$ seguido de alimentación, apoyo emocional, y sexualidad, la de menor porcentaje es la actividad física con 70,1\%, estas prácticas afectan y ponen en riesgo a la persona que vive con VIH.

En la tabla 3, el 70,1\% no presenta adherencia según los resultados del instrumento de evaluación; el 29,9\% presenta adherencia es decir cumplen con el TARGA.
Tabla 3. Perfil de adherencia al TARGA de las personas que viven con VIH de un hospital nacional. Octubre-diciembre 2013, Lima-Perú

\begin{tabular}{lcc}
\hline Perfil de adherencia & n & \% \\
\hline Adherencia & 86 & 29,9 \\
No adherencia & 202 & 70,1 \\
Total & 288 & 100,0 \\
\hline
\end{tabular}

Tabla 4. Correlación entre prácticas de autocuidado y adherencia en un hospital nacional. Octubre-diciembre 2013, Lima-Perú

\begin{tabular}{|c|c|c|c|c|}
\hline \multirow{2}{*}{$\begin{array}{l}\text { Prácticas de } \\
\text { autocuidado }\end{array}$} & \multicolumn{2}{|c|}{ Perfil de adherencia (CD4) } & \multirow{2}{*}{ Total } & \multirow{2}{*}{$\begin{array}{c}\text { Rho de } \\
\text { Spearman }\end{array}$} \\
\hline & Adherencia & No adherencia & & \\
\hline \multirow{2}{*}{ Adecuadas } & 0 & 1 & 1 & $0,445^{*}$ \\
\hline & $0,0 \%$ & $100,0 \%$ & $100,0 \%$ & $\mathrm{P}=0,000^{*}$ \\
\hline \multirow{2}{*}{ Inadecuadas } & 86 & 201 & 287 & \\
\hline & $30,0 \%$ & $70,0 \%$ & $100,0 \%$ & \\
\hline \multirow{2}{*}{ Total } & 86 & 202 & 288 & \\
\hline & $29,9 \%$ & $70,1 \%$ & $100,0 \%$ & \\
\hline
\end{tabular}

* Estadísticamente significativa

En la tabla 4, el coeficiente de correlación rho de Spearman evidencia una correlación significativa $(p=0,000)$ entre las variables prácticas de autocuidado y adherencia. El valor del coeficiente de correlación rho de Spearman $(\mathrm{R}=0,445)$ muestra una correlación media $\mathrm{y}$ positiva, lo que significa que a niveles altos de prácticas de autocuidado le corresponden niveles altos de nivel de adherencia, o viceversa.

\section{DISCUSIÓN}

Las estadísticas de morbi y mortalidad, a nivel mundial, en personas portadoras de. virus de inmunodeficiencia humana se han incrementado considerablemente. El Perú no ha sido la excepción, por ello, se estima un tratamiento integral y especializado de dicha enfermedad, considerando que los obstáculos existentes pueden ser la persona misma o los agentes del cuidado, como el equipo de salud (1).

En los resultados se puede observar que el 99,7\% tiene prácticas inadecuadas, mientras el $0,3 \%$ presenta prácticas adecuadas; esto puede deberse a falta de madurez para asumir con responsabilidad el autocuidado. Valverde manifiesta que la falta de difusión e internacionalización del autocuidado en la persona, dificulta asumir una postura de adherencia al programa del TARGA, y que se prescriba normas de conducta difícilmente practicables y sostenibles genera, a menudo, frustración y sentimientos de culpabilidad que contribuyen a la falta de interés por el autocuidado. Por lo tanto, es importante describir las dimensiones de las prácticas del autocuidado para conocer específicamente los comportamientos de las personas que viven con VIH (7).

Respecto a la alimentación, el 90,3\% (260) muestra que no tiene una adecuada práctica alimentaria, de ello se deduce la importancia de la alimentación para fortalecer el sistema inmune y lograr el mejoramiento en la calidad de vida. Cardona $y \mathrm{col}$. refieren que la alimentación es la base para mantener un buen recuento de linfocitos $\mathrm{CD} 4$, condicionar la progresión de portador asintomático o sintomático a SIDA, mejorar el sistema inmune y facilitar la acción de los antirretrovirales. Por otra parte, Orem considera que el autocuidado es la madurez que logra el individuo y la familia para lograr participación y adaptación a la situación de salud que lo aqueja, esto contribuye a generar conductas de promoción y prevención de las enfermedades oportunistas que aparecen en esta enfermedad $(8,5)$. 
Pennington muestra que el $100 \%$ de los usuarios que viven con VIH, de la unidad de atención integral, identifican fácilmente los temas relacionados con la alimentación, lo que confirma que la educación nutricional que se da cumple con su objetivo principal (9).

La persona que vive con VIH necesita satisfacer sus requerimientos alimenticios para mantener un nivel óptimo de su sistema inmune que le permitirá lograr condiciones adecuadas para una mejor adherencia al TARGA y, a la vez, disminuir la posibilidad de contraer enfermedades oportunistas. Pero, los alimentos que consumen estas personas en casa, no cumplen muchas veces con las condiciones sanitarias para contrarrestar la enfermedad, ello debido a diversos factores propios de su idiosincrasia y cultura y al no aceptar su propia enfermedad. En muchos casos desconocen la calidad de los alimentos que deben consumir, además, no se cumple con el manejo y la conservación adecuados por lo que se presentan efectos secundarios como diarreas, vómitos, continuas bajas de peso, etc. (9).

Otro punto que considerar es la higiene, en este estudio se muestra que el 94,1\% (271) no practica el lavado de manos antes de consumir alimentos y después de ir al baño, esto demuestra la falta de autocuidado lo cual coloca al afectado muy vulnerable a la aparición de enfermedades oportunistas. El MINSA, en su proyecto «Cerrando brechas hacia el logro de los objetivos del milenio en TBC y VIH en el Perú», refiere que la higiene es una necesidad básica que garantiza la práctica de autocuidado, las condiciones de las personas, en relación con la higiene, se ven condicionadas por la educación, los patrones culturales, los factores socioeconómicos, etc.; de lo antes mencionado se deduce que la persona no es un ente aislado, ni separada de sí mismo, tiene que cuidarse holísticamente, de tal manera que todo su proceso de atención esté enfocado en su curación, en su desarrollo y su crecimiento como persona (10), la higiene marca la pauta para una mejor adherencia y una mejor armonía de la persona con su tratamiento puesto que el cuidado del cuerpo, favorece las buenas prácticas de autocuidado y mejora el sistema inmune.

Según Varela, la calidad de vida de las personas que viven con VIH, está determinada por la noción del autocuidado, proceso identificado con la enfermedad o su diagnóstico. Realmente ,la visión holística de la propia vida, de una persona aseada y limpia, marca la pauta y la diferencia, porque una persona que se cuida y que mantiene una buena, permanente higiene y limpieza, mejora su estado inmune (11).
En cuanto a la sexualidad, se observa que 79,5\% (229) tiene prácticas inadecuadas, puesto que no usan preservativos, lo cual coincide con los resultados de Juárez $\mathrm{J} y$ col., quienes afirman que las personas que viven con $\mathrm{VIH}$ y reciben TARGA parecen tener comportamientos sexuales de riesgo y estarían facilitando la transmisión del virus a sus parejas; también está de acuerdo con lo encontrado en estudios epidemiológicos de naturaleza cuantitativos, que afirman que el inicio de la era TARGA está relacionada con la continuación o incremento de comportamientos sexuales de riesgo entre hombres homo y bisexuales (12).

Dos razones podrían explicar por qué las personas que viven con VIH y que reciben TARGA tienen comportamientos sexuales de riesgo. En primer lugar existe la creencia de que al producirse el «adormecimiento» del virus por acción de los antirretrovirales, no hay peligro. El virus del VIH «adormecido» si se trasmite a una persona sana, en todo caso, dependerá del estilo de vida de esta persona para que desarrolle la infección. En segundo lugar, hay la creencia que cuando el personal de salud les comunica que tienen carga viral indetectable, ellos consideran que han sido curados de la infección. Estas creencias, probablemente, los llevan a tener prácticas inadecuadas, pues el ser personas que viven con VIH, compromete a tener una sexualidad responsable y estilos de vida saludables, además, esto lo fundamenta el Instituto de Medicina Tropical Alexander Von Humboldt, cuando menciona que la evolución de la enfermedad requiere de condiciones favorables (13).

La capacidad de afrontamiento que tienen los pacientes con VIH, depende muchas veces de la aceptación de su enfermedad y de los estilos de vida saludable, incluyendo el autocuidado. Además, la situación de falta de voluntad y de no aceptación de la enfermedad está ampliando la cantidad de contagios a nivel mundial.

El apoyo emocional es inadecuado en un 85,1 \% (245), porque no viven su vida con total tranquilidad y no aceptan su enfermedad, estos resultados coinciden con el estudio de Cantú $y$ col. quienes afirman que en el soporte emocional y social se percibió la ausencia de apoyo, centrándose básicamente en lo económico y laboral; la persona que recibe un resultado de ELISA positivo pasa por un momento de desesperanza y tristeza profunda, y es allí donde se requiere soporte emocional para darle la oportunidad de recuperar la salud, motivarle a superar el estigma y cambiar su estilo de vida (14).

En este apoyo, relacionado con las emociones y sentimientos, se debe ofrecer un importante respaldo, para 
conseguir que la persona cumpla con el tratamiento y con la asistencia puntual a sus controles permanentes y así mantenerlos cautivos en el proceso continuo de educación. La persona debe tener confianza para expresar sus sentimientos e inquietudes, esto ayudará a trabajar los posibles efectos adversos y no correr el riesgo que pueda atentar contra su propia vida, o perjudique la salud de otros individuos. Ante esto, es importante educar, para asegurar el autocuidado y que se proteja la confidencialidad del diagnóstico. Con relación al descanso, se le explica a la persona que el sueño contribuye a mejorar el estado inmune y el no aprender a canalizar las emociones, perturba el sueño, altera el estado de ánimo y perjudica su salud. Es importante trabajar en el estado emocional, por lo prolongado de la enfermedad y las reacciones adversas a los medicamentos; un buen acompañamiento durante el proceso de la enfermedad ayudará a superar el duelo que conlleva la aceptación, esto permitirá al agente de salud brindar un buen apoyo emocional y al cumplimiento del tratamiento.

En lo referente a la actividad física, este estudio muestra que el $70,1 \%$ la ejecuta inadecuadamente debido a que no realizan caminatas para obtener un óptimo estado de salud según indicación médica, datos similares a los hallados por Cardona (8); por otro lado, Benito (15) describe que el ejercicio terapéutico puede aumentar la capacidad funcional de trabajo, la fuerza y la resistencia muscular, al tiempo que contribuye a revertir la pérdida muscular y los estados de adelgazamiento mórbido. La actividad física ayuda a las personas con VIH a sentirse mejor, porque fortalece su sistema inmune y revitaliza su estado psicológico; es recomendable que la persona realice todo tipo de acciones físicas, pero sin excederse, para no llegar a innecesarias deshidrataciones, que podrían ser perjudiciales para mantener fluidos corporales en sus niveles normales, por ello, se sugiere el seguimiento médico; Cardona (8) concluye que el ejercicio no afecta negativamente al estado inmune en lo que respecta al recuento de células cd4 o carga viral, no se observaron cambios significativos $y$, en todo caso, la tendencia era hacia el aumento en el recuento de linfocitos cd4, entre los que realizaban ejercicios en comparación con los controles sin ejercicios (12), (14), (15).

El autocuidado cumple una función importante en los estilos de vida de las personas con VIH, esto está fundamentado por la teórica Dorotea Orem, quien describe que la persona es un todo que funciona biológicamente, simbólicamente y socialmente y que posee las capacidades, las aptitudes y la voluntad de comprometerse y de llevar a cabo autocuidados, estos son de tres tipos: los autocuidados universales, los asociados a los procesos de crecimiento y de desarrollo humano y los unidos a desviaciones de salud. Por ello, la persona que vive con VIH, requiere de autocuidados en el área de actividad física (5).

Piña col., afirman que los factores de la adherencia relacionada al paciente pueden ser: alimentos, vivienda, vestido, transporte, horarios y trabajo. Para reducir el impacto negativo de estos factores, es necesario realizar una evaluación detallada de los factores mencionados, utilizar recursos comunitarios disponibles, apoyarlos o crearlos, citas en horarios flexibles, y facilitar la comunicación con un agente de soporte, ya sea la pareja, un familiar, amigos o algún miembro de la comunidad (16).

La enfermera dentro del programa del TARGA, administra los autocuidados y ejecuta el sistema de enfermería de apoyo-educación, además, interviene ayudando a las personas para que sean competentes de realizar su propio autocuidado y lograr la madurez de autocuidarse de la mano con el soporte familiar.

En este estudio se aprecia que el 70,1\% no cumple con el nivel de adherencia al TARGA, es decir, la persona no está concientizada con su enfermedad, evade responsabilidades con respecto a la toma de sus medicamentos en los horarios establecidos; a mayor prácticas de autocuidado, mayor adherencia, pero solo el 29,9\% alcanza una buena adherencia. Andrade nos explica que la prevalencia de abandono del TARGA en lima es 4 veces mayor que en provincia $(4,09 / 0,98)$ y que el $81 \%$ de los abandonos ocurren en Lima. Por lo tanto, la adherencia al TARGA cumple un papel clave al garantizar la efectividad de los fármacos, habiéndose demostrado que una incorrecta adherencia se relaciona con el aumento de los ingresos hospitalarios.esta realidad contrasta con Peñarrieta, quien observó que la tasa de no adherencia fue del 50 y el $48 \%$, en los últimos cuatro días y cuatro semanas, respectivamente (17), (18).

Ceccato refiere que aquellas personas con ingesta del $95 \%$ o más del total de comprimidos prescritos por día, eran clasificados como seguidores del tratamiento. El seguimiento fue de $73,3 \%$ y el objetivo del estudio fue medir la prevalencia por falta de seguimiento al tratamiento antirretroviral altamente eficaz en personas con sida. El acompañamiento de las personas que viven VIH requiere de un seguimiento permanente para mantener sus prácticas de autocuidado (19).

la gestión del cuidado de enfermería tiene como objetivo fundamental, favorecer el cuidado integral, las prácticas 
de autocuidado y la adherencia, constituye una necesidad de crear conciencia en las personas que viven con VIH para adoptar comportamientos de autocuidado y el acompañamiento de una capacitación educativa sostenida donde se debe incorporar la interacción enfermero - persona que viven con VIH. Es importante resaltar, que la medicación debe darse de manera sostenida y programada, sino fuera asi esta puede obstaculizar los beneficios de salud de la persona. Conjuntamente, una mala adherencia puede conllevar a la aparición de resistencias y a la progresión de la infección por VIH al estado de sida, con mucha mayor rapidez.

Alvis, en perú, considera que los factores independientemente asociados a la no adherencia fueron: ser homosexual/bisexual, tener una baja calidad de vida relacionada a la salud, poco apoyo social, no tener domicilio fijo, tener morbilidad psíquica, tener mayor tiempo en tratamiento y los factores de tipo psicosocial, fueron los que influyeron de forma más importante en la falta de adherencia al tratamiento antirretroviral. De igual modo, Muralles expresa que es necesario restablecer, mantener $\mathrm{y}$ fortalecer el sistema inmune para favorecer la adherencia, esta se encuentra estrechamente ligada al tratamiento y las mantener prácticas de autocuidado (20), (21).

Por ello, las personas enfermas que inician el tratamiento deben ser consecuentes con toda la medicación establecida, tomarlas en los horarios ya programados, porque el darle continuidad a este requerimiento, los sitúa como personas adherentes al tratamiento y los resultados serán más efectivos, es importante resaltar en el marco de la terapia integral, que si no se utiliza la medicación de manera sostenida y programada, esta puede limitar y mermar sus beneficios, una mala adherencia puede conllevar a la aparición de resistencias y a la progresión de la infección por VIH al estado de sida. Todo lo mencionado lo reafirma Varela et al. en su estudio enuncia que la persona puede abandonar el tratamiento por diferentes circunstancias, por algunas reacciones adversas y otros por mala adherencia, de esto se deduce, que los problemas de adherencia son sumamente importantes y las cifras reales constituyen una necesidad de seguir explorando a esta población (11).

Finalmente Guelar col., en su estudio de estrategias para optimizar la adherencia al tratamiento antirretroviral, relata que antes de iniciar el tratamiento, se debería estar preparado en los tres niveles de respuesta humana: cognitivo, conductual y emocional, esto requiere información, habilidades y motivación; para ello, es necesario información básica para conocer las causas por las que se debe iniciar el tratamiento (niveles de linfocitos cd4 y carga viral); el modo de actuación del tratamiento; las implicaciones que representa en la vida diaria; la importancia de seguir fielmente la posología; la duración indefinida; los riesgos que supone no iniciar, y el riesgo de una adhesión inadecuada, porque la educación busca generar y favorecer una mejor calidad de vida, aunque el resultado anhelado es lograr la madurez en la aplicación de la práctica de autocuidado (22).

En cuanto a la relación de la práctica del autocuidado y adherencia con el nivel de adherencia al TARGA, el coeficiente rho de Spearman $(\mathrm{r}=0,445)$ muestra una correlación significativa $(\mathrm{p}=0,000)$, la cual resultó positiva y de nivel medio, lo que significa que niveles altos de prácticas de autocuidado se corresponden con niveles altos de adherencia y viceversa.

La relación entre prácticas de autocuidado y nivel de adherencia es significativa para este estudio, pues se llega a la conclusión de que existe una correlación fuerte y altamente significativa, esto quiere decir, que cuanto mayor sean la práctica, mayor es el nivel de adherencia. Al igual que estudios antes mencionados, coinciden en la misma apreciación y describen que las personas que viven con $\mathrm{VIH}$, requieren de mejores condiciones físicas, biológicas, estabilidad emocional y elevación de la autoestima.

De otros estudios relacionados con prácticas de autocuidado y adherencia al TARGA se puede evidenciar que los problemas de adherencia se vinculan esencialmente con la insatisfacción de las necesidades básicas de las personas que viven con VIH y que el manejo responsable de buenas prácticas de autocuidado y la fidelidad al tratamiento del TARGA favorecerán las condiciones óptimas de salud, porque crearán una buena adherencia. Aquí podemos mencionar algunos:

Varela col., refieren que la persona puede abandonar el tratamiento por diferentes circunstancias, unos por algunas reacciones adversas, otros por mala adherencia. Asimismo, Ballester concluye que existen cambios significativos en el grado de adhesión al tratamiento de las personas, variables clínicas relevantes como la ansiedad, la depresión o la autoestima y otras como el grado de hipocondría y perturbación afectiva (11), (23).

Los estudios concluyen que se debe lograr crear, diseñar y plasmar nuevas y mejores estrategias, para beneficiar e implementar a los servicios de enfermería, tanto en relación con las prácticas de cuidado y en su parte educativa. Pero este es un trabajo en conjunto con las diferentes disciplinas de salud, cuyo único objetivo central tiene que 
ser el bienestar de la persona, sin olvidar nunca que a mayor y mejor autocuidado, mayor y mejores posibilidades de adherencia al tratamiento antirretroviral.

La persona que vive con VIH, ante todo, debe disponer de información adecuada, esto significa que desde el inicio al tratamiento se debe concientizar y educar para incentivar la correcta forma de la administración de la terapéutica integral que consiste en el control, seguimiento y la evaluación periódica del perfil de adherencia (de los niveles de linfocitos CD4 y carga viral), además de ello, seguir fielmente la posología, tomando en cuenta que la duración es indefinida, y las prácticas de autocuidados en su vida cotidiana deben generar condiciones favorables para disminuir los riesgos de enfermedades oportunistas, asimismo, el no iniciar correctamente el tratamiento integral lo coloca en riesgo a una adhesión inadecuada (creación de resistencias y fracaso terapéutico).

La no adherencia al tratamiento es una problemática de muchos países desarrollados y subdesarrollados, tiene causas o factores que dificultan la buena adherencia, entre ellos tenemos los problemas para integrar el cumplimiento al tratamiento, mientras la persona que vive con VIH por sentimiento de discriminación mantenga en reserva el diagnóstico dificulta la interacción social y familiar entre otras actividades diarias o las responsabilidades que se tengan para con otras personas como hijos, parientes a cargo, etc., y problemas que impiden cumplir con los requisitos que la medicación que exige muchas veces establece con respecto a las comidas entre otras situaciones que afecta su vulnerabilidad.

Muralles menciona que los factores que facilitaron la adherencia fueron los tratamientos con menos unidades por dosis al día; la recuperación del sistema inmune es mayor en las personas adherentes desde el principio de los tratamientos y con valores de adherencia por arriba del $90 \%$, los efectos adversos a los medicamentos (sobre todo los digestivos), tuvieron una repercusión directa en el porcentaje de adherencia de los pacientes (21).

La población que participó en este estudio tiene las siguientes características sociodemográficas: el $68,1 \%$ es de sexo masculino; el $47,6 \%$ son solteros y el $34,4 \%$ son convivientes. Esto nos muestra que los varones tienen una alta tasa para el riesgo de la infección de VIH, determinándose que los solteros y convivientes son variables, condicionante para el riesgo de la infección y exposición al virus del VIH. Estos datos coinciden con los estudios epidemiológicos del MINSA, donde se demuestran la alta tasa de incidencia de infección de VIH en el sexo mascu- lino, condicionándose la vulnerabilidad de la «opción sexual» y/o «orientación sexual», que muestra el sexo masculino.

Cabe señalar que de los 288 encuestados, 205 no participan en el grupo GAM (grupo de ayuda mutua) esta organización se encarga de sensibilizar y promover a las personas con la enfermedad del VIH/SIDA para que aprenda a conocerse y compartir experiencias de autocuidado a otras personas que están en la misma situación, por lo tanto, si la persona se excluye, se encuentra en posible riesgo a la no adherencia al TARGA, condicionado a las alteraciones fisiológicas y psicológicas de las personas infectadas.

Finalmente, es importante resaltar la importancia de educar sobre las prácticas de autocuidado y la adherencia del TARGA en las personas que viven con VIH, el objetivo es beneficiar a estas personas que acuden en búsqueda de mejorar su salud y los programas educativos deben procurar seguir las pautas de conductas que afiancen el autocuidado y que puedan ayudar al individuo a mantener su salud. Para el profesional de enfermería, es valorar el proceso integral terapéutico que beneficie el cuidado y la prolongación de vida de la persona. La teoría del autocuidado es imprescindible para fundamentar el desarrollo de la misma, además de integrarla como parte de una necesidad que demanda la adherencia. Sumado a esto, la consejería es de vital importancia para implementar y desarrollar programas educativos, que contemplen aspectos dirigidos a concientizar a la persona sobre la importancia de adoptar prácticas de autocuidado, desde el inicio del tratamiento del TARGA, para mejorar sus condiciones de salud futuras.

Se concluye que las prácticas de autocuidado de las personas que viven con VIH son inadecuadas casi en su totalidad (99,7\%). Según las cinco dimensiones de las prácticas de autocuidado son inadecuadas en porcentajes elevados: higiene $94,1 \%$; alimentación $90,3 \%$; apoyo emocional $85,1 \%$; sexualidad $79,5 \%$ y actividad física $70,1 \%$.

\section{REFERENCIAS BIBLIOGRÁFICAS}

1. Organización Panamericana de la Salud. Experiencias Exitosas en el Manejo de la Adherencia al Tratamiento Antirretroviral en Latinoamérica [Internet] 2011. (citado 12 Ene 2011). Disponible en: http:// w w w . p a h o.org/h q/i n d ex.ph p ? option=com_docman\&task $=$ doc_view\&gid $=23855 \&$ Itemid $=$ 
2. Porras Campos J. VIH- SIDA: intervenciones para mejorar la adherencia. UPCH, Instituto de medicina tropical Alexander von Humboldt, 2da. Ed. 2004.

3. MINSA. Cuidados domiciliarios para personas que viven con VIH/SIDA; 3 ed. 2005 - 2010.

4. MINSA. Recomendaciones para implementar un programa antirretroviral en Lima- Perú; 2004.

5. Marriner T. Modelos y Teorías en Enfermería. $7^{\mathrm{a}}$ ed. España: Elsevier Sciencie; 2011.

6. Colegio de Enfermeros del Perú y Consejo Nacional.» Normas de gestión de la calidad del cuidado enfermero», Lima - Perú; 2008.

7. Valverde R. Saber para vivir o saber morir? Apropiación de información sobre autocuidado en personas que viven con VIH. En: Cáceres F. y colaboradores, editor. La salud sexual como derecho en el Perú de hoy. Estudios sobre salud, género y derechos sexuales entre los jóvenes, otros grupos vulnerables. Perú: REDES JOVENES; 2002: 181-07.

8. Cardona J, Peláez J, Saldarriaga D, Molina L. Revista: Cielo: Calidad de vida de pacientes con VIH/SIDA y atención integral de enfermería. Scielo. 16(4):2. (citado 13 Feb 2013). Disponible en: http://scielo.i ics.una.py/scielo.php?pid=S199636962010000 100004\&script $=$ sci_arttext

9. Pennington J. Elaboración de material de educación alimentaria nutricional como apoyo a proveedores/as de salud que brindan consejería a personas adultas no alfabetos que viven con VIH.Guatemala.2010

10. Ministerio de Salud. Proyecto cerrando brechas hacia el logro de los objetivos del milenio en TBC y VIH en el Perú; Módulo de entrenamiento en consejería en prevención positiva y adherencia al TARGA para profesionales de salud en Lima-Perú: MINSA; 2007.

11. Varela $\mathrm{M}$, et al. Adherencia al tratamiento en la infección por VIH-SIDA. Consideraciones teóricas y metodológicas para su abordaje. Acta Colombiana de Psicología 2009.

12. Juárez J, Pozo E. Percepciones sobre compor tamientos sexuales de riesgo en personas que viven con VIH/SIDA y reciben tratamiento antirretroviral en Piura, Perú. (citado 20 Ene 2013); 10 (2):2encontrado http://www.uv.mx/psicysal2/Rodrigo $\%$ 20Cant $\%$ FA\%20Guzm\%E1n_2.pdf

13. Adherencia y resistencia al TARGA [Internet]. Lima: $\mathrm{UPCH}$, Instituto de medicina tropical Alexander von Humboldt, Jáuregui; 2004 (citado 5 Mar 2011). URL disponible en: http://www.upch.edu.pe/tropicales/ telemedicinatarga/MODULOS/ADHERENCIA.htm

14. Cantú G, Álvarez B, Torres E, Martínez O. Impacto psicosocial en personas que viven con VIH-sida, México:2012 (citado 25 Abr 2013); 16(4):2- encontrado http://www.uv.mx/psicysa12/ Rodrigo\%20Cant\%FA\% 20Guzm\%E1n_2.pdf

15. Benito G, María E, Efectos del ejercicio físico en adultos con VIH/SIDA: revisión sistemática.Madrid.2012

16. Piña JA, Sánchez-Sosa JJ, et al. Un Modelo psicológico en los comportamientos de adhesión terapéutica en personas con VIH, Redalyc.Org; 2009.

17. Andrade L. Revisión de la adherencia al TARGA y directrices para su mejoramiento tras 4 años de tratamiento ARV en Perú. Noviembre 2008

18. Peñarrieta MI. Experiencia de vivir con el VIH / SIDA en un país latino: México: Tamaulipas; 2006

19. Ceccato M. Factores de riesgo para la no-adherencia al tratamiento con terapia anti-retroviral altamente eficiente. 2008.

20. Alvis O, De Coll L, Chumbimune L, Díaz C, Díaz J, Reyes M. Factores asociados a la no adherencia al tratamiento antirretroviral de gran actividad en adultos infectados con el VIH-sida. Lima-Perú. 2009.

21. Muralles O. La adherencia a tratamientos con medicamentos anti-retrovirales en pacientes que acuden a la clínica familiar «Luis Ángel García» del hospital general san Juan de Dios. Guatemala: 2005.

22. Guelar A, Knobel H. Estrategias para optimizar la adherencia al tratamiento antirretroviral. Hospital del Mar. Barcelona. España. 2003.

23. Ballester R. Eficacia terapéutica de un programa de intervención grupal cognitivo-comportamental para mejorar la adhesión al tratamiento y el estado emocional de pacientes con infección por VIH/ SIDA.2003.

24. Goenaga N. Evaluación de la Adherencia al Tratamiento en Pacientes con VIH Sida.2009.

25. Adaptación al cuestionario de evaluación de la adhesión al tratamiento antirretroviral (CEAT- VIH) para su uso en Perú An Fac med. 2009;70(4):266-72 [Internet]. 2008. (citado16 Abr 2013). URL disponible en http://farmacia.ugr.es/ars/pdf/434.pdf.

26. ONUSIDA. Programa Conjunto de las Naciones Unidas sobre el VIH/SIDA. Informe sobre la Epidemia Mundial del SIDA. ONUSIDA; 2011.

27. Cáceres F, et al. Promoción de la salud sexual: Aportes para la Investigación y la Acción. Perú: Instituto de Estudios en Salud, Sexualidad y Desarrollo Humano - IESSDEH; 2009.

28. Smeltzer S, Bare B. Enfermería Médico Quirúrgico.12 ed. Madrid: Mc. Graw Hill Interamericana; 2013.

29. Ministerio de Salud de Salud, Dirección General de Epidemiología. Análisis de la Situación Epidemiológica del VIH/SIDA en el Perú, 2013. 
30. Guía de enfermería para la atención de las personas con VIH. Consejo Nacional para la Prevención y Control del SIDA CONASIDA. México DF.2012.

31. Marguilles S, Barber N, Recoder ML. VIH-SIDA y «adherencia» al tratamiento. Enfoques y perspectivas Antípoda. Revista de Antropología y Arqueología [Internet] 2006, (julio-diciembre): (citado 14 Oct 2012) Disponible en: <http://www.redalyc.org/ articulo.oa? id=81400313 $>$ ISSN 1900-5407.

32. Peñarrieta, DCI. Adherencia al tratamiento antirretroviral en personas con VIH. [tesis]. México: Tamaulipas; 2008.

\section{Correspondencia}

Mónica Meneses La Riva

Universidad Cesar Vallejo

Correo electrónico:mmeneseslariva@yahoo.com

Forma de citar este artículo: Meneses-La Riva M, MayorcaCarmelo C. Prácticas de autocuidado y la adherencia al TARGA en personas que viven con VIH en un hospital nacional de Lima. Rev enferm Herediana. 2014;7(2):87-96. 